\title{
Brain Tumor Detection from MR Images using Image Process Techniques and Tools in MATLAB Software
}

\author{
Faisal Rehman, Syed Sheeraz Shah, Hamadullah Panhwar, Akhtar Hussain, Sajjad Ali, Shuaib \\ Hussain, Tarique Mehmood, Saeed Rabbani
}

\begin{abstract}
In the medical era the Brain tumor is one of the most important research areas in the field of medical sciences. Researcher are trying to find the reliable and cost effective medical equipment's for the cancer and its type for the diagnosed, especially tumor has deferent kinds but the major two type are discussed in this research paper. Which are the benign and Pre-Malignant, this research work is proposed for these factors such as the accuracy of the MRI image for the tumor identification and actual placing were taken into consideration. In this study, an algorithm is proposed to detect the brain tumor from magnetic resonance image (MRI) data simple. As enhance the image quality for the easiness the tumor treatments and diagnosed for the patients. The proposed algorithm enhances the MR image quality and detects the Brain tumor which helps the Physician to diagnose the tumor easily. As well this algorithm automatically calculates the area of tumor, size and location of the tumor where it is present for diagnostic the Patient.
\end{abstract}

Keywords: MRI-Simples, Classification, Brain Tumor, Detection, Image Process Technique and Tools.

\section{INTRODUCTION}

The body is made up of many types of cells each type of cell has special function. Most of the cells in the body grownup and then divide in an orderly way to form new cells as they are needed to keep the body healthy as the body is working properly. When the cells lose the ability to control their growth, they divide too often and without any order. In the medical filed the extra cells form a mass or tissues in body that is called the tumor. Basically Tumors has different kind but in my research work, I have mentioned two kind of tumor

\footnotetext{
Manuscript received on 10 July 2021 | Revised Manuscript received on 17 July 2021 | Manuscript Accepted on 15 August 2021 | Manuscript published on 30 August 2021.

* Correspondence Author

Faisal Rehman*, National University of Science of Technology Islamabad. Email: thebo_Faisal@yahoo.com

Syed Sheeraz Ali, Sukkur IBA University Sindh Pakistan. Email sheraz.ali@iba-suk.edu.pk

Hamadullah Panhwar, Jilin University PR China Email: hamadali78@yahoo.com

Dr. Akhtar Hussain Phul, Khairpur Medical College KMC Khairpur. Email: theradiologist74@gmail.com

Sajjad Ali Rajpar, Mohammad Ali Jinnah University Sindh Pakistan Email: sajjad.ali.se13@iba-suk.edu.pk

Shuaib Ahmed, Kunming University of Science and Technology, China. Saeed Rabbani, Sukkur IBA University.

Tarique Mehmood, Quaid Awam University Sindh.

(C) The Authors. Published by Lattice Science Publication (LSP). This is an open access article under the CC-BY-NC-ND license (http://creativecommons.org/licenses/by-nc-nd/4.0/)
}

One is benign or malignant and other is Pre-malignant. There are three methods of segmentation such as Snakes Gradient Vector Flow (GVF), Level Set the Segmentation as well as the Watershed Segmentation process [1].

The aim of this study is to design an automated tools or devices for brain tumor quantification of tumor, classification and identification of tumor cells by using MRI image data simple sets. This work is a small and modest part of a quite complex system for the image process, the whole system will on completion visualize the inside of the human body, and enable surgeons to perform operations on a patient without open surgery or other test performances. More specifically, this work is to images segmenting a tumor in a brain. This kind of work will help the surgeon to detect the tumor and then provide and ease for the treatment of tumor.

The instruments needed for this could be Ultrasound, Computer Tomography (CT scan) and Magnetic Resonance Imaging (MRI). These technique adopted in this study is Magnetic Resonance Imaging (MRI) and used the MATLAB image tools to test the brain tumor images simples.

The MRI is an imaging technique that produces high quality images of the anatomical structures of the human body, especially in the brain, and provides rich information for clinical diagnosis and biomedical research [2]-[6]. The diagnostic values of MRI are greatly magnified by the automated and accurate classification of the MRI images [7]-[9]. Wavelet transform is an effective tool for feature extraction from MR brain images, because it allows analysis of images at various levels of resolution due to its multi-resolution analytic property. However, this technique requires large storage and is computationally expensive [10]. In order to reduce the feature vector dimensions and increase the discriminative power, the principal component analysis (PCA) was used [11]. In the literature review we have gone through suggested proposed scheme for the image quality enhancement in the mentioned research work some of the research are proposed filtration methods for the image quality techniques like as a Noise removal using wiener filter, leaner contrast adjustment, Median filtration and the decorrelation stretch filtration techniques [12].

The PCA is appealing since it effectively reduces the dimensionality of the data and therefore reduces the computational cost of analyzing new data [13]. Then, the problem of how to classify the input data arises. In recent years, several researchers have proposed different approaches to achieve this goal, which fall into two categories.

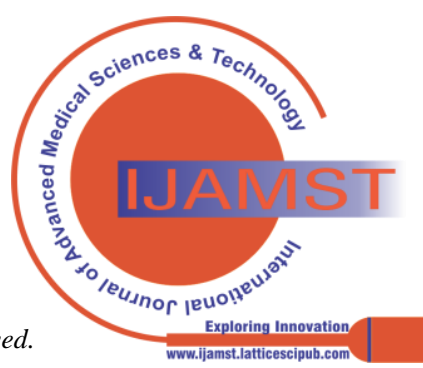


One category is supervised classification, including support vector machine (SVM) and $\mathrm{k}$ nearest neighbors (k-NN).

The other category is unsupervised classification including self-organization feature map (SOFM) and fuzzy c-means. While all these methods achieved good results, and yet the supervised classifier performs better than unsupervised classifier in terms of classification accuracy rate for the image (success classification rate).

The researcher and biomedical devices manufacturing companies are trying to develop and design the cost effective equipment's for this kind of disease identification and detection as well as the diagnosed the patients. However, one of the of the big challenges are to image quality and its detection of tumor in recently few research has found the accuracy and image quality near to $94 \%$. So we have try to overcome these factor and get near to $96 \%$ images quality and its classification but over proposed scheme has some minor issue, the overall results are more accurate and as compare to the pervious mentioned schemes.

\section{EXPERIMENTAL RESULTS AND DISCUSSION}

This research work is based on image processing method and filter quality for the MRI image for the detection of the tumor from brain and used these methods to detection and identification of the tumor from the Magnetic Resonance Imaging (MRI) data simples. The MRI images are used as a simple for the real time application to detect the tumor cells in the human body, but the most of the machine capture the these images are less quality and low resolutions so we have design the algorithm that will first capture the data and then apply the filters to enhance or improve the images quality and then segments the images for the tumor detection and in final it will classified the tumor type and display the results, for the enhancement of image quality and then detection of the tumor. The two sections involved in this study are tumor detection and identification of tumor like a Benign and Pre-tumor. First, MRI image used as simple and the proposed algorithm is applied on trained algorithm for the image process. The proposed algorithm enhances the image quality by using filters and then removes the noise from the MRI images. After that it applies the segmentation process to detect the tumor and identify the tumor type as shown in Table 1 and Table 2, respectively. This algorithm is just take few second for the process the images to classification and detection the tumor as mentioned in the results sections.

In this research work, we have consider two case for this research one is benign tumors and other pre-benign tumor as shown in the In Table 1. There are two kind of samples were taken for the experiment, that is, shown in Table 1, 2. And shown in Figures (a\&b), there are two samples of MRI images are tested in this algorithm. The proposed algorithm first will remove the additional Noise by using the leaner filtrations techniques, after that it will apply the methods of segmentation in the last I will classified the tumor type in first case have use only one type of cancer images.

As in the above Table1 only designed algorithm is tested benign tumor simple in this portion algorithm achieved the image quality accuracy is $98 \%$ and the overall algorithm performance is $96 \%$ in term of classification, reliability and time parameters ,the images are the (2 and 2). When tumor is detected, then, this algorithm identifies the tumor type and produce the final results as given in Table.1.

Table 1 Brain Tumor Type Benign Detection

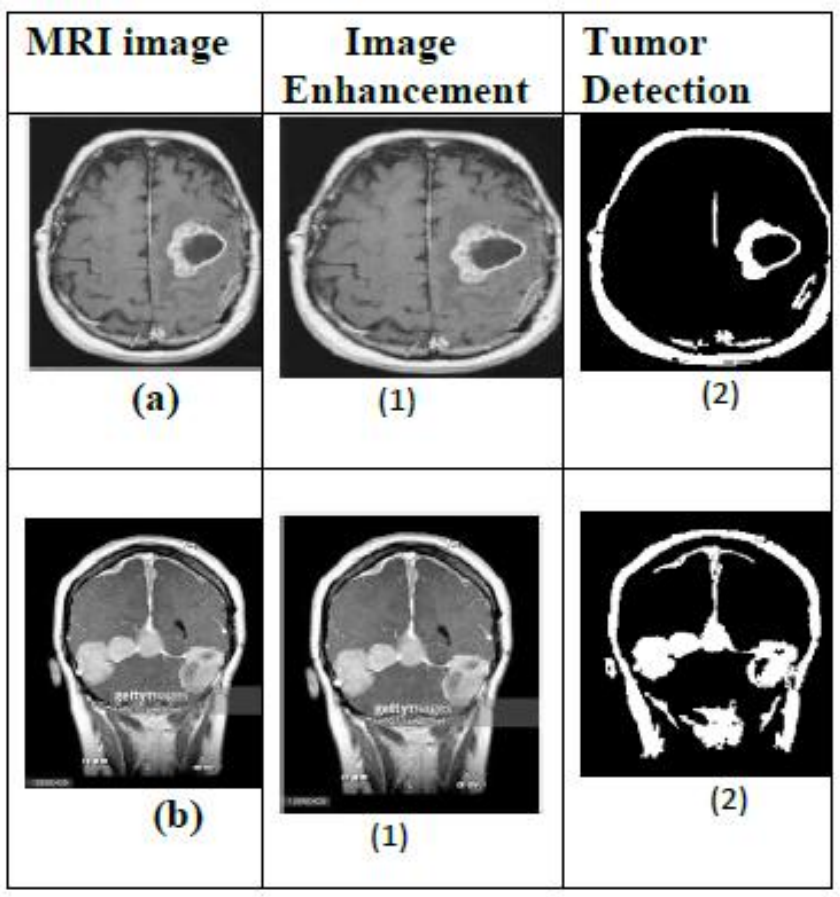

As shown in the table-2 for the second type tumor detection from the trained MR Images as the setup are same for this type of the process to test the algorithm except the tumor type, as in in the figure (a\&b) was taken after and before the testing ad figure $(1 \mathrm{a}, 2 \mathrm{a}$ and $\mathrm{b} 1, \mathrm{~b} 2)$. These two samples of the brain tumor are taken with the help of the MRI Machine. These MR images has low resolution and its big issue for the physician to treatment the cancer patients, the designed algorithm is used to remove the additional noise with the leaner filtration tolls and show the high quality regulations for the furthermore process, the second part is to segmented the loaded images in algorithm and then classified the images as for the detection and identification of the tumor. As shown in given in Table 2.in the final results images are the (a2 and b2) showing the tumor classification and detection and also shown in table-1. All the process finishing tumor is detected, then, this algorithm identifies the tumor type like as in table-2 data is pre-malignant and in table- 1 for the benign type of tumor. Overall results are more accurate and having accuracy of $96 \%$ and less time taken for the both cases as well as the other cases and applicable for the CT and MR scan image date and testing.

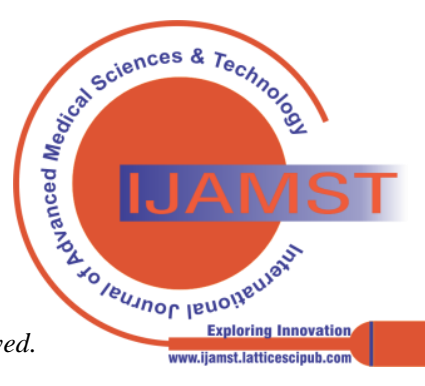


Table 2 Brain tumor Pre-Maligned Detection

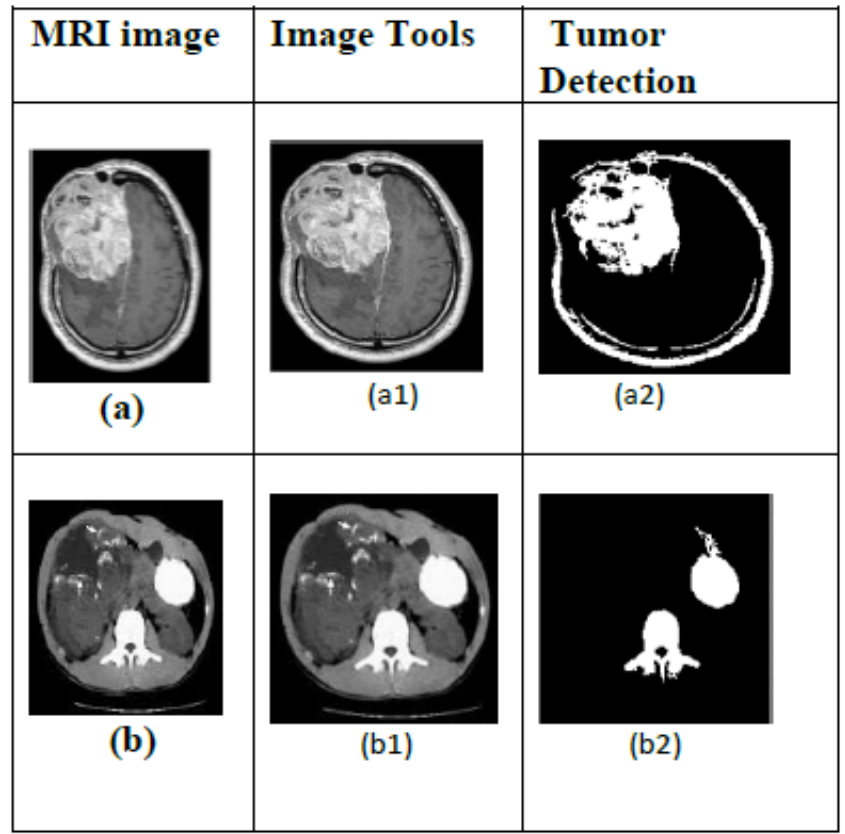

\section{CONCLUSION}

This research work is proposed to detect the tumor type from the MR Images simples and to identify, detection of brain tumor with the help of the image process tools and techniques. So we have developed new the algorithm for the image quality improve, enhancements and used the segmentation techniques for the classification and identifications of the tumor types with the help of the proposed algorithm. The proposed algorithm first removes the noise from the images and then enhances the quality of the images and subsequently classified the images for detections of the tumor from the MR simple in the final it is declare the tumor type and identification.

This research work will helps out the physicians to conveniently diagnose the tumor Patients and this research clearly show the tumor size, location and where it is present, so the physicians can easily save the patients from the disease and provide them better treatments for the better life. This research work has got more significant results in term of the accuracy, enhancement and less processing time and give the more efficient results for the diagnosed applications the overall results accuracy level is $96 \%$ in term of the time and detection of the tumor from the CT and MR images, this algorithm is can apply for the any MR and CT scan machine for the significant results with high accuracy and easiness for the treatments.

\section{REFERENCES}

1. Abbasi,S and Mokhtarian, F. Affine-similar Shape Retrieval: Application to Multi view 3-D object Recognition. 131-139. IEEE Trans, Image processing vol.10, no. 1, -2001. [CrossRef]

2. Zhang, Y., L.Wu, and S. Wang, Magnetic resonance brain image classification by an improved artificial bee colony algorithm," Progress In Electromagnetics Research, Vol. 116, 65-79, 2011. [CrossRef]

3. Mohsin, S. A., N. M. Sheikh, and U. Saeed, MRI induced heating of deep brain stimulation leads: Effect of the air-tissue interface," Progress in Electromagnetics Research, Vol. 83, 81-91, and 2008. [CrossRef]
4. Golestanirad, L., A. P. Izquierdo, S. J. Graham, J. R. Mosig, and C. Polo, Effect of realistic modeling of deep brain stimulation on the prediction of volume of activated tissue," Progress In Electromagnetics Research, Vol. 126, 1-16, 2012. [CrossRef]

5. Mohsin, S. A, Concentration of the specific absorption rate around deep brain stimulation electrodes during MRI," Progress in Electromagnetics Research, Vol. 121, 469-484, 2011. [CrossRef]

6. Oikonomou, A., I. S. Karanasiou, and N. K. Uzunoglu,Phasedarray near field radiometry for brain intracranial applications, " Progress In Electromagnetics Research, Vol. 109, 345-360, 2010. [CrossRef]

7. Scapaticci, R., L. Di Donato, I. Catapano, and L. Crocco, A feasibility study on microwave imaging for brain stroke monitoring," Progress In Electromagnetics Research B, Vol. 40,305-324, 2012. [CrossRef]

8. Asimakis, N. P., I. S. Karanasiou, P. K. Gkonis, and N. K. Uzunoglu, Theoretical analysis of a passive acoustic brain monitoring system," Progress In Electromagnetics Research B, Vol. 23, 165-180, 2010. [CrossRef]

9. Chaturvedi, C. M., V. P. Singh, P. Singh, P. Basu, M. Sing ravel, R. K. Shukla, A. Dhawan, A. K. Patti, R. K. Gangwar, and S. P. Singh.2.45 GHz (CW) microwave irradiation alters circadian organization, spatial memory, DNA structure in the brain cells and blood cell counts of male mice, muss musculus," Progress In Electromagnetics Research B, Vol. 29, 23-42, 2011. [CrossRef]

10. Emin Tagluk, M., M. Akin, and N. Sezgin, Classification of sleep apnea by using wavelet transform and artificial neural networks," Expert Systems with Applications, Vol. 37, No. 2, 1600-1607, 2010. [CrossRef]

11. Zhang, Y., L. Wu, and G. Wei, A new classifier for polar metric SAR images," Progress in Electromagnetics Research, Vol. 94, 83- 104, 2009. [CrossRef]

12. Kushwaha, S., \& Singh, R. K. (2015). Study and analysis of various image enhancement method using MATLAB. image, 7, 8.

13. Camacho, J., J. Pico, and A. Ferrer, Corrigendum to 'the best approaches in the on-line monitoring of batch processes based on PCA: Does the modelling structure matter?' [Anal. Chim. Act Volume 642 (2009) 59-68]," Analytical Chemical Act.

\section{AUTHORS PROFILE}

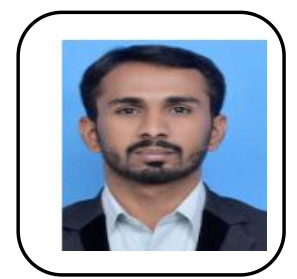

Faisal Rehman, Mr. F.Rehman is doing his Post Gradation in Lab on Chip form the Yangzhou University of Science China, His area of research is Bioelectronics, Lab on Chip, Material Technology, and Membrane fabrication of Graphene, Go Oxide, and Electronic Fabrication Design, Image process, Biomedical Engineering WBSNs, Mr FR Profile has 18 research articles that published in Books, Journals.

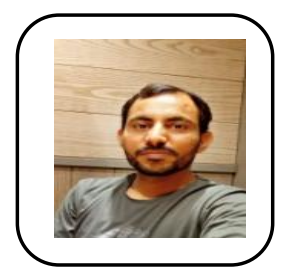

Syed Sheeraz Shah Mr Sheeraz is currently doing his MS in electronic Communication from Jiangsu University of china, His area of interest is Biomedical Engineering, Electronics Communication, and Computer Networking.

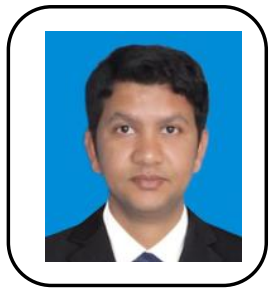

Hamadullah Panhwar, Mr Hamad did his bachelor degree in Yangtze University, Hubei, and did my Master degree in Changchun University of Technology, and currently he doing his $\mathrm{PhD}$ from Jilin University, Jilin Province China his research area is Electronics system Design, Biomedical Sensors, Image Process, Material Science and Piezoelectric Devices. 


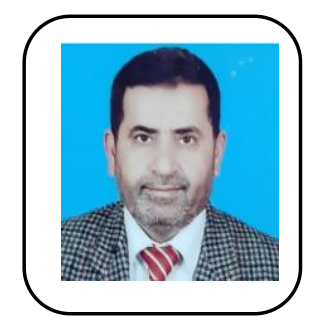

Dr Akhtar Hussain, Dr Akhtar Hussain Phul Associate Professor Diagnostic Radiology Department of Khairpur Medical College Khairpur Sindh Pakistan, Dr Hussain Research area is Radiological Process, CT scan, MRI and Ultrasonic, X-rays based Disease Detection and its Treatment's,

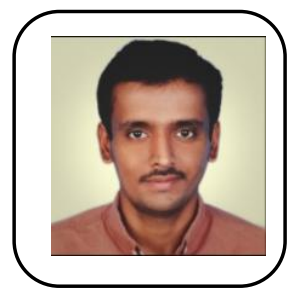

Sajjad Ali Rajpar, Mr Rajpar is Doing His MS in Computer Science Form Mohammad Ali Jinnah University Sindh Pakistan. His area of research is Biomedical Images, MRI Images, CT scan, Image Process and Block chain, Data Mining for the Medical Processing.

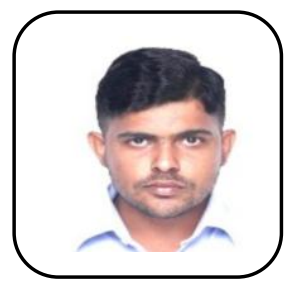

Shuaib Hussain, Shuaib is currently doing MS Electronics Form the Kunming University of Science and Technology, PR China His area is Electronics System design, Boost Converter, Sensors, Image Processing and thermocouple devices.

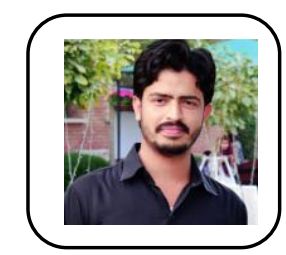

Tarique Mehmood, Mr. Mehmood is Electrical Engineer Currently Doing his ME in Electronic from the Quaid Awam University NawabShah. His research area is Electronic, Biomedical Image Process, WBSNs, and Wireless Endoscopy.

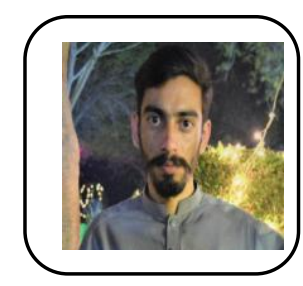

Saeed Rabbani, Saeed is Doing Bachelor of science in Computer Science from Sukkur IBA University.

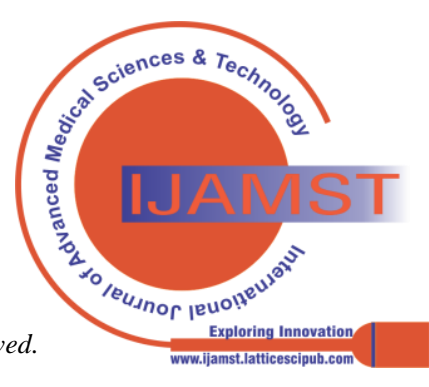

